\title{
一种三足葱三唑化合物的合成及其对录离子荧光识别
}

\author{
朱江华范香田蒋平曹迁永* \\ (南昌大学化学系 南昌 330031)
}

\begin{abstract}
摘要 通过点击反应合成了一个以苯为中心含葱荧光团的三足三唑受体分子 1. 在 $\mathrm{CH}_{3} \mathrm{CN}-\mathrm{H}_{2} \mathrm{O}(V: V=9: 1)$ 溶液中, 受体分子 1 对采离子表现出很好的苂光淬灭响应. 其荧光淬灭率为 $75 \%$, 对录离子的检测极限为 $6.1 \times 10^{-6} \mathrm{~mol} / \mathrm{L}$. ${ }^{1} \mathrm{H}$ $\mathrm{NMR}$ 滴定表明受体分子中三唑环中的三个 $\mathrm{N}$ 原子与中心离子参与了配位.
\end{abstract}

关键词 三足受体; 三唑; 葱; 录离子; 苂光识别

\section{A Tripodal Anthracene-Appended Triazole for Fluorescence Sensing of $\mathrm{Hg}^{2+}$}

\author{
Zhu, Jianghua Fan, Xiangtian Jiang, Ping Cao, Qianyong* \\ (Department of Chemistry, Nanchang University, Nanchang 330031)
}

\begin{abstract}
A new anthracene-containing tripodal triazole with 1,3,5-trisubstituted benzene core has been easily synthesized by "click reaction". Among various metal ions, 1 shows exclusive fluorescence turn-off sensing of $\mathrm{Hg}^{2+}$ in $\mathrm{CH}_{3} \mathrm{CN}-\mathrm{H}_{2} \mathrm{O}(V: V=$

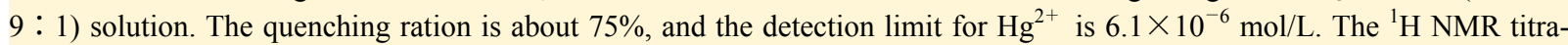
tions results reveal that the $\mathrm{N}$ atoms of the three triazoles of 1 take part in binding with center ion with forming $1: 11-\mathrm{Zn}^{2+}$ complex.
\end{abstract}

Keywords tripodal receptor; triazole; anthracene; mercury ion; fluorescence sensing

采及其化合物具有很高的生物毒性 $[1,2]$, 在人体内 富集会引发多种疾病, 如产前脑损伤及水俣病. 因此开 发能够快速检测人体和环境中录具有重要的意义. 苂光 光谱法由于其操作简便、灵敏度高、检测速度快等优点, 能快速、高效地检测微量的录离子, 得到人们的广泛关 注. 苂光光谱法由于好的敏感性与选择性、较低的成本、 信号容易检测等优点在录离子识别领域占有重要的地 位 ${ }^{[3 \sim 24]}$. 目前, 基于不同光调控机理识别录离子的苂光 化学传感器已有大量文献报道, 但其中大部分易受其他 金属离子如 $\mathrm{Ag}^{+} 、 \mathrm{Cu}^{2+}$ 和 $\mathrm{Cd}^{2+}$ 的干扰 ${ }^{[21 ~ 24]}$. 因此, 研究 对录离子具有高选择性和灵敏度的新型化学传感器仍 具有挑战性.

铜催化的叠氮化物与末端炔点击反应生成的 $1,2,3-$ 三唑化合物近年来在超分子化学领域受到人们广泛关 注 ${ }^{[25,26]}$. 这种点击反应不仅是一种有效的共价键连接基 团, 同时生成的三唑环中 3-位 $\mathrm{N}$ 原子可与金属离子配
位. 通过合理的化学修饰, 用于金属离子识别 ${ }^{[27 ~ 29]}$. 一 些基于三唑苂光受体用于识别已有相关文献报道 ${ }^{[18 ~ 23]}$. 另一方面，含有多个金属离子结合位点的三足受体分子 也得到了很大的关注 ${ }^{[30]}$. 这种三足受体由于具有特殊 的三维立体结构和更多的结合位点, 当分析物与三足受 体所构成的空腔尺寸与形状匹配时, 一般会具有较强的 结合能力. 基于此, 一些通过点击反应得到的三足三唑 受体用于金属离子识别的已经报道. 其中一个众所周知 受体为三(1-苠基-[1,2,3]-三唑-4-亚甲基)胺(TBTA), 对 过渡金属和重金属离子具有很强的结合能力 ${ }^{[31,32]}$, 且它 的铜络合物用于催化点击反应 ${ }^{[33]}$. Ingale 等 ${ }^{[34]}$ 报道过芘 修饰三足(三唑)胺化合物, 在乙腈溶液中, 可对锌离子 实现比率荧光响应. Sambri 等 ${ }^{[35]}$ 报道过三足(三唑) $\mathrm{Ce}^{3+}$ 配合物, 用于蓝光材料. Molina 等 ${ }^{[36]}$ 报道了基于 $1,3,5$ 取 代苯为中心含萠苂光团三足(三唑)受体，可用于 $\mathrm{Hg}^{2+}$, $\mathrm{Cu}^{2+}$ 和 $\mathrm{Pd}^{2+}$ 的荧光识别. 近期笔者等 ${ }^{[37]}$ 报道葱修饰三

\footnotetext{
* Corresponding author(s). E-mail: cqyong@ncu.edu.cn

Received June 23, 2016; revised July 25, 2016; published online September 1, 2016.

Project supported by the National Natural Science Foundation of China (Nos. 21162017, 21462027).

国家自然科学基金(Nos. 21162017, 21462027)资助项目.
} 
足(三唑)胺化合物, 在乙腈一水溶液中可荧光识别 $\mathrm{Ni}^{2+}$.

本文报道一种通过 “点击” 反应获得了一种以苯作 为骨架含葱苂光团三足三坐化合物 1 (Eq. 1), 但研究其 金属离子识别性质. 在乙腈-水 $(V: V=9: 1)$ 溶液中, 受体 1 对 $\mathrm{Hg}^{2+}$ 有很好的苂光淬灭响应, 且不受其它阳离 子的干扰.

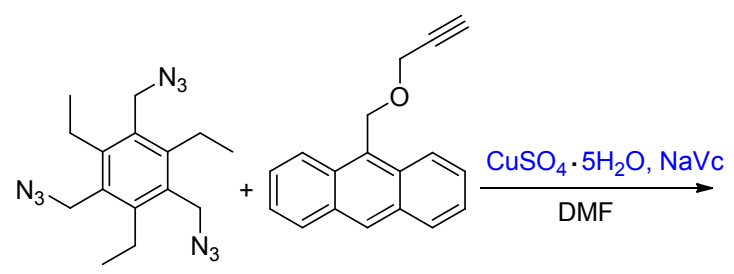

2

3

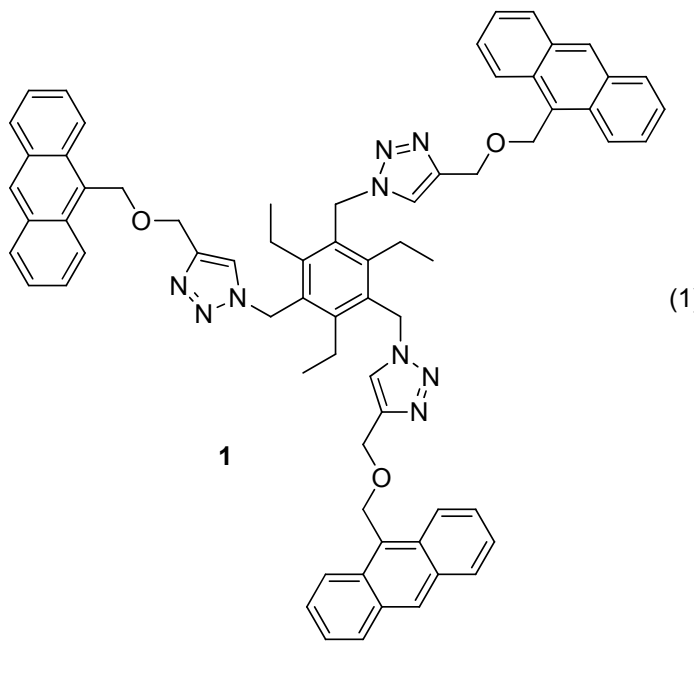

Yield: $62 \%$

\section{1 结果与讨论}

\section{1 合成目标化合物研究}

化合物 1 的合成见 Eq. 1. 以 1,3,5-三乙基苯及 9-葸 甲基醇为原料, 首先根据文献方法 ${ }^{[38,39]}$ 合成出 $2,4,6$-三 叠氮甲基-1,3,5-三乙基苯(2)和 9-[(2-炔丙氧基)甲基] 葱 (3). 然后以 $\mathrm{CuSO}_{4} / \mathrm{NaVc}$ 为催化剂, 在 $\mathrm{DMF}$ 溶液中等量 化合物 2 与 3 通过 “点击” 反应得到三足三唑受体 $\mathbf{1}$. 目标产物通过 ${ }^{1} \mathrm{H}$ NMR, ${ }^{13} \mathrm{C}$ NMR 及 ESI-MS 得到证实. 在 ${ }^{1} \mathrm{H}$ NMR 中, 三唑环上的氢位于 $\delta 7.95$ 单峰 $\left(\mathrm{CDCl}_{3}\right.$ 溶 液), 与相关文献一致 ${ }^{[36]}$.

\section{2 阳离子的苂光光谱研究}

我们首先通过 UV-vis 光谱测定化合物 1 在 $\mathrm{CH}_{3} \mathrm{CN}-\mathrm{H}_{2} \mathrm{O}(V: V=9: 1)$ 溶液中对金属离子 $\left(\mathrm{Na}^{+}\right.$, $\mathrm{Ca}^{2+}, \mathrm{Mg}^{2+}, \mathrm{Ba}^{2+}, \mathrm{Co}^{2+}, \mathrm{Fe}^{2+}, \mathrm{Al}^{3+}, \mathrm{Cr}^{3+}, \mathrm{Ag}^{+}, \mathrm{Cu}^{2+}, \mathrm{Ni}^{2+}$, $\mathrm{Zn}^{2+}, \mathrm{Cd}^{2+}, \mathrm{Hg}^{2+}, \mathrm{Pd}^{2+}$ 和 $\mathrm{Pb}^{2+}$ )识别能力. 化合物 1 在 345,365 和 $385 \mathrm{~nm}$ 处出现了三个吸收峰, 可归属为生色
团葸的 $\pi-\pi *$ 跃迁．当滴加不同金属离子时，受体分子 1 仅对 $\mathrm{Hg}^{2+}$ 有很好的吸收光谱响应. 图 1 为受体分子 $\mathbf{1}$ 中 滴加不同浓度 $\mathrm{Hg}^{2+}$ 时 UV-Vis 光谱变化图, 从图中可以 看出, 随着 $\mathrm{Hg}^{2+}$ 浓度增加, 受体分子 1 中基于葸的吸收 峰减弱且红移 $(\approx 2 \mathrm{~nm})$. 此外, 在滴定过程中, 在 350 , 368 和 $388 \mathrm{~nm}$ 处可观察到三个等吸收点, 表明 1 和 $\mathrm{Hg}^{2+}$ 之间存在唯一结构的络合作用.

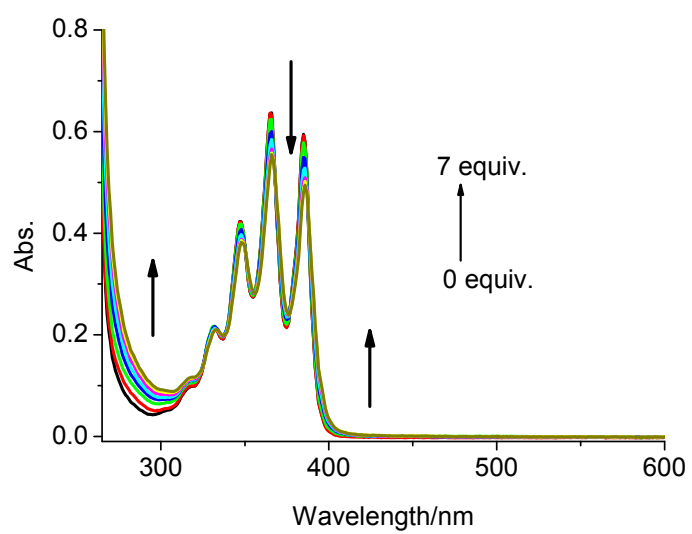

图 $1 \mathrm{CH}_{3} \mathrm{CN}-\mathrm{H}_{2} \mathrm{O}$ 中 $(V: V=9: 1)$ 滴加 $\mathrm{Hg}^{2+}(0 \sim 7$ equiv. $)$ 时受 体 $1\left(2.0 \times 10^{-5} \mathrm{~mol} / \mathrm{L}\right)$ 的紫外-可见吸收光谱变化图

Figure 1 UV-vis spectra of $1\left(2.0 \times 10^{-5} \mathrm{~mol} / \mathrm{L}\right)$ upon addition various amount of $\mathrm{Hg}^{2+}\left(0 \sim 7\right.$ equiv.) in $\mathrm{CH}_{3} \mathrm{CN}-\mathrm{H}_{2} \mathrm{O}(V: V=$ $9: 1)$ solution.

接下来研究受体 $\mathbf{1}$ 对上述金属离子的荧光识别能力 (图 2). 以 $360 \mathrm{~nm}$ 为激发波长，受体 1 在 390,412 和 435 $\mathrm{nm}$ 处有强的吸收峰, 可归属为蒽荧光团的单体发射. 当添加 $\mathrm{Hg}^{2+}$ 时，受体 $\mathbf{1}$ 表现出极强的苂光淬灭响应，其 荧光淬灭率达到 $75 \%$ (加入 10 equiv. $\mathrm{Hg}^{2+}$ ). 这种荧光响 应表明受体 $\mathbf{1}$ 与 $\mathrm{Hg}^{2+}$ 发生了强的络合作用, 受体 $\mathbf{1}$ 中葱 环的激发态与中心离子 $\mathrm{Hg}^{2+}$ 之间发生了光诱导电子转 移(PET)而导致受体分子荧光淬灭效应，如同一些文献 中所报道 ${ }^{[18,19]}$. 相反, 当添加其他金属离子, 受体 $\mathbf{1}$ 的 苂光几乎没有变化, 这与紫外-可见吸收光谱结果一致. 虽然基于 $\mathrm{Hg}^{2+}$ 的荧光探针报道很多，但很多受体分子 常受到 $\mathrm{Ag}^{+}$与 $\mathrm{Cu}^{2+}$ 等金属离子干扰. 受体分子 $\mathbf{1}$ 表现出 的 $\mathrm{Hg}^{2+}$ 专一性识别, 将表现出更好的应用前景.

通过化合物 $\mathbf{1}$ 与 $\mathrm{Hg}^{2+}$ 苂光光谱滴定，进一步定量评 估受体 1 和金属离子之间的结合能力(图 3). 随着 $\mathrm{Hg}^{2+}$ 浓度的增加, 化合物 1 在 $412 \mathrm{~nm}$ 处苂光强度逐渐减少. 当滴加 10 equiv. 的 $\mathrm{Hg}^{2+}$ 时, 受体 $\mathbf{1}$ 的荧光发射光谱强度 达到饱和. 通过苂光滴定线性拟合得出 $\mathbf{1}$ 和 $\mathrm{Hg}^{2+}$ 结合的 化学计量学为 $1: 1$. 根据 Benesi-Hildebrand ${ }^{[40]}$ 方程计算 出 1 对 $\mathrm{Hg}^{2+}$ 的络合常数为 $2.7 \times 10^{3}{\mathrm{~L} \cdot \mathrm{mol}^{-1}}^{-1}(R=0.993$, 图 4a), 对 $\mathrm{Hg}^{2+}$ 检测限为 $6.1 \times 10^{-6} \mathrm{~mol} / \mathrm{L}(R=0.993$, 图 4b). 


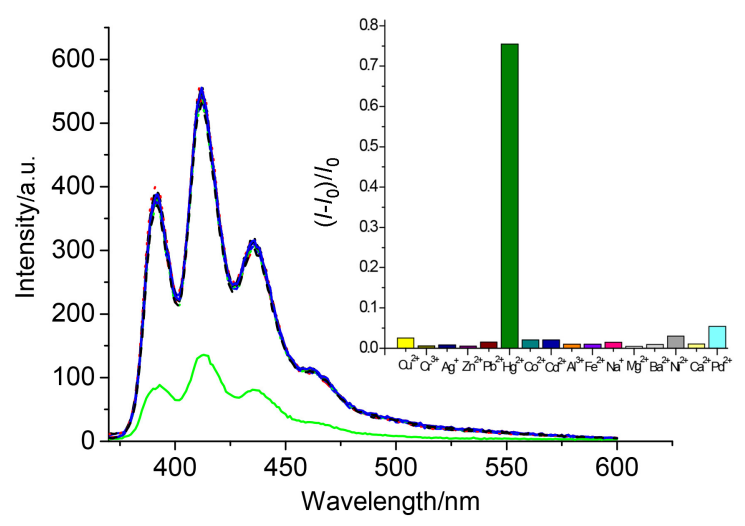

图 2 在 $\mathrm{CH}_{3} \mathrm{CN}-\mathrm{H}_{2} \mathrm{O}(V: V=9: 1)$ 中滴加 10 倍量不同金属离 子时受体 $1\left(2.0 \times 10^{-5} \mathrm{~mol} / \mathrm{L}\right)$ 的苂光发射光谱图

Figure 2 Emission spectra of $1\left(2.0 \times 10^{-5} \mathrm{~mol} / \mathrm{L}\right)$ upon addition of 10 equiv. metal ions in $\mathrm{CH}_{3} \mathrm{CN}-\mathrm{H}_{2} \mathrm{O}(V: V=9: 1)$ solution

Inset: the quenching ratio bar of $\mathbf{1}$ at $412 \mathrm{~nm}$ with addition various metal ions

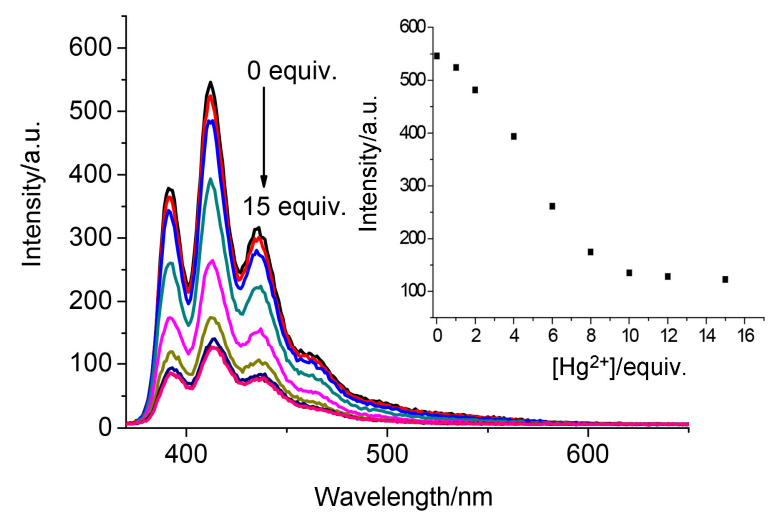

图 3 在 $\mathrm{CH}_{3} \mathrm{CN}-\mathrm{H}_{2} \mathrm{O}(V: V=9: 1)$ 溶液中滴加 $\mathrm{Hg}^{2+}$ 化合物 1 的苂光光谱变化图(激发波长为 $360 \mathrm{~nm}$ )

Figure 3 Emission spectra of 1 upon the addition of various of $\mathrm{Hg}^{2+}$ in $\mathrm{CH}_{3} \mathrm{CN}-\mathrm{H}_{2} \mathrm{O}(V: V=9: 1)$ solution

Inset: the fluorescence intensity of $\mathbf{1}$ at $412 \mathrm{~nm}$ versus the equivalence of $\mathrm{Hg}^{2+}$

为了进一步研究受体 $\mathbf{1}$ 对 $\mathrm{Hg}^{2+}$ 的选择性识别, 我们 做了 1 中加入 $\mathrm{Hg}^{2+}$ 及其它共存金属离子 $\left(\mathrm{Na}^{+}, \mathrm{Ca}^{2+}\right.$, $\mathrm{Mg}^{2+}, \mathrm{Ba}^{2+}, \mathrm{Co}^{2+}, \mathrm{Fe}^{2+}, \mathrm{Al}^{3+}, \mathrm{Cr}^{3+}, \mathrm{Ag}^{+}, \mathrm{Cu}^{2+}, \mathrm{Pd}^{2+}, \mathrm{Zn}^{2+}$, $\mathrm{Cd}^{2+}$, 和 $\mathrm{Pb}^{2+}$ )的竞争性实验(图 5). 结果表明, 其他金属 离子的存在对于 $1-\mathrm{Hg}^{2+}$ 络合物的苂光并没有发生显著 变化, 表明在乙腈-水溶液中, 化合物 1 对 $\mathrm{Hg}^{2+}$ 有很好 的苂光选择性识别.

\section{3 识别机理研究}

为了进一步理解受体分子 $\mathbf{1}$ 与 $\mathrm{Hg}^{2+}$ 的络合机制, 我 们以 $\mathrm{CD}_{3} \mathrm{CN} / \mathrm{CDCl}_{3}(V: V=2: 8)$ 为溶剂研究了受体分 子 1 中滴加 $\mathrm{Hg}^{2+}$ 时 ${ }^{1} \mathrm{H} \mathrm{NMR}$ 变化. 从图 6 可以看出, 当 滴加 $\mathrm{Hg}^{2+}$ 时, 受体分子 $\mathbf{1}$ 中的三唑环质子 $\mathrm{H}_{\mathrm{a}}$ 从 $\delta 7.41$
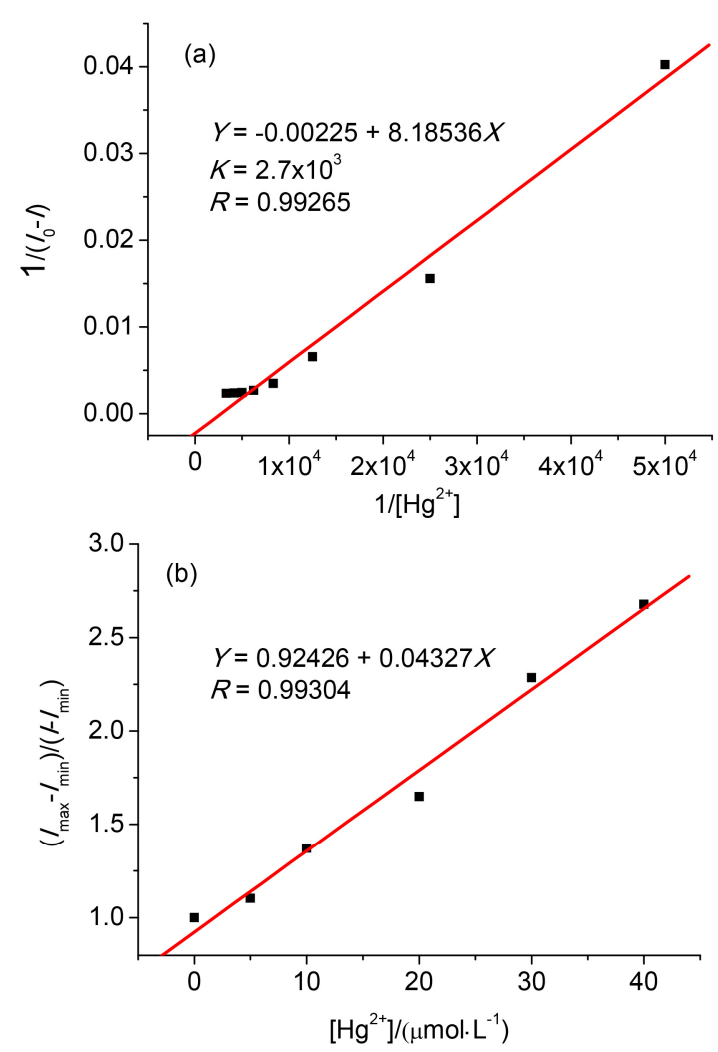

图 4 在乙腈的水溶液中受体 $1-\mathrm{Hg}^{2+}\left(2 \times 10^{-5} \mathrm{~mol} / \mathrm{L}\right)$ 的 Benesi-Hildebrand 直线 (a) 和 1 对 $\mathrm{Hg}^{2+}$ 的检测限(b)

Figure 4 Benesi-Hildebrand plot for $1: 1$ complexation of 1 with $\mathrm{Hg}^{2+}$ (a) and the detection limit (b) of $\mathbf{1}$ for $\mathrm{Hg}^{2+}$ according to the emission data

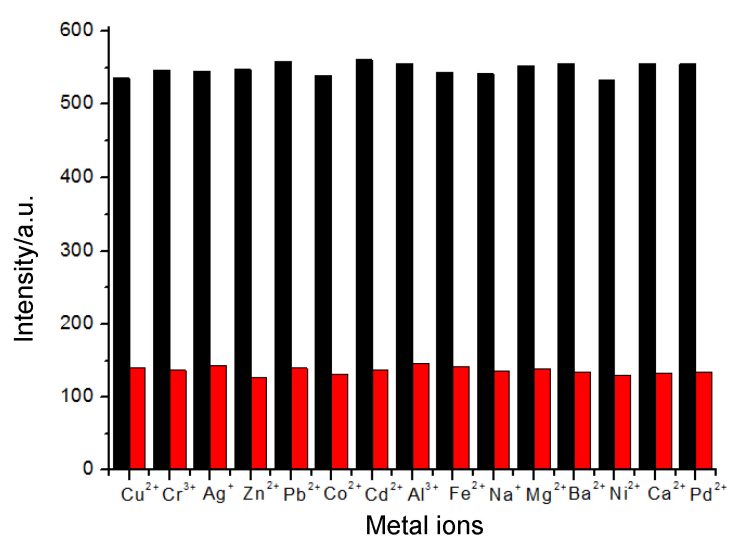

图 5 在 $\mathrm{CH}_{3} \mathrm{CN}-\mathrm{H}_{2} \mathrm{O}(V: V=9: 1)$ 溶液中受体 $1\left(2.0 \times 10^{-5}\right.$ $\mathrm{mol} / \mathrm{L}$ )中滴加 10 倍量不同金属离子(前)再滴加 $\mathrm{Hg}^{2+}$ (后)412 $\mathrm{nm}$ 荧光强度变化

Figure 5 Emission changes of $1(20 \mu \mathrm{mol} / \mathrm{L})$ at $412 \mathrm{~nm}$ upon addition 10 equiv of other metal ions (front) and then addition $\mathrm{Hg}^{2+}$ in $\mathrm{CH}_{3} \mathrm{CN}-\mathrm{H}_{2} \mathrm{O}(V: V=9: 1)$ solution (behind)

移动至 $\delta 7.53$ 处，表明三唑上的氮原子参与了和 $\mathrm{Hg}^{2+}$ 的 络合作用. 同时，三条三唑臂中的亚甲基质子 $\mathrm{H}_{\mathrm{b}} 、 \mathrm{H}_{\mathrm{c}}$ 与 $\mathrm{H}_{\mathrm{d}}$ 随着受体 $\mathbf{1}$ 与 $\mathrm{Hg}^{2+}$ 的络合发生了一定程度的高场移 


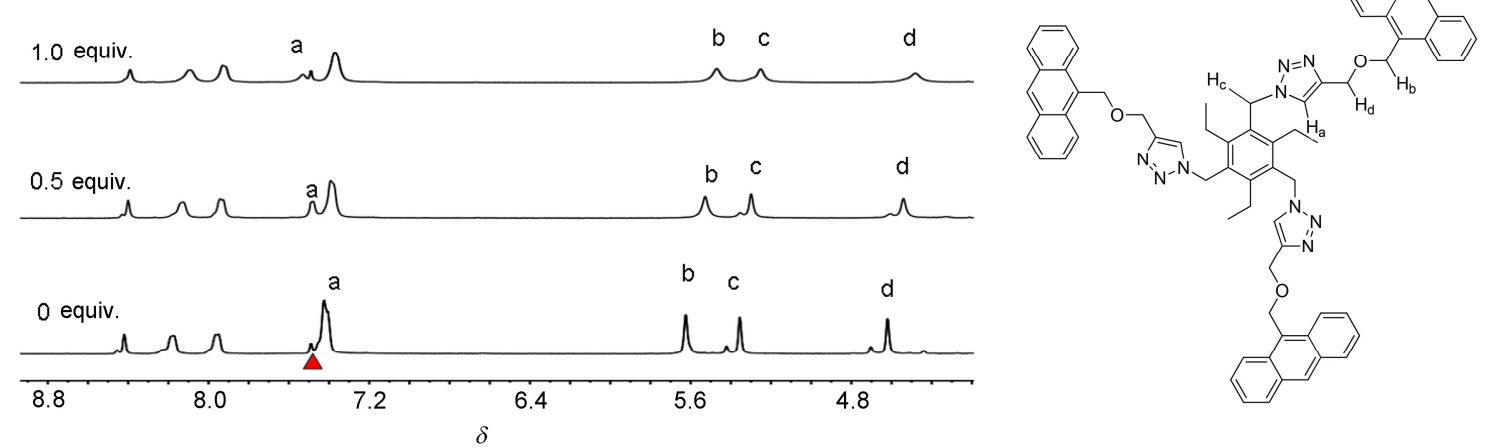

图 6 在 $\mathrm{CD}_{3} \mathrm{CN}-\mathrm{CDCl}_{3}(V: V=2: 8)$ 中受体 1 滴加 $\mathrm{Hg}^{2+}$ 时核磁氢谱变化滴定 $(\boldsymbol{\Delta}$ 为氞代氯仿溶剂峰 $)$

Figure $6{ }^{1} \mathrm{H}$ NMR spectrum of 1 upon addition of $\mathrm{Hg}^{2+}$ in $\mathrm{CD}_{3} \mathrm{CN}-\mathrm{CDCl}_{3}(V: V=2: 8)$ solution $\left(\boldsymbol{\Delta}\right.$ denotes the residual $\mathrm{CDCl}_{3}$ peak)

动, 其移动值分别为 $\delta 0.15 、 0.11$ 及 0.14 , 这可能归因于 受体分子与 $\mathrm{Hg}^{2}$ 络合后三条柔性臂旋转受到限制的结 果. 类似受体分子络合刚性增强导致的质子高场移动在 文献中已有报道 ${ }^{[36,41]}$.

\section{2 结论}

以 2,4,6-三叠氮甲基-1,3,5-三乙基苯和 9-[(2-炔丙氧 基)甲基]葱为原料点击反应合成了一个以苯为中心含葱 苂光团的三足三坐受体分子 1 , 并研究了其对金属离子 识别能力. 结果表明, 在乙腈含水溶液中, 受体分子 $\mathbf{1}$ 对 $\mathrm{Hg}^{2+}$ 有很好的专一性识别, 且不受其他金属离子干 扰. 随着 $\mathrm{Hg}^{2+}$ 的加入, 受体分子 $\mathbf{1}$ 的苂光发生了 $75 \%$ 的

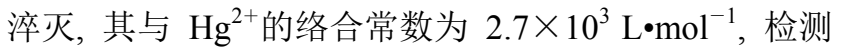
极限为 $6.1 \times 10^{-6} \mathrm{~mol} / \mathrm{L}$. ${ }^{1} \mathrm{H}$ NMR 滴定表明受体 1 的三唑 环上的 3 个 $\mathrm{N}$ 原子与中心离子发生了配位作用.

\section{3 实验部分}

\section{1 仪器与试剂}

核磁共振仪(德国 Bruker 公司, Bruker Vance 400 MHz, TMS 为内标)、飞行时间质谱仪(美国 Waters 公司 XEVO G2 QTof 型)、元素分析仪(德国 Elementar 公司 Vario EL III 型)、紫外可见光谱(Hitachi 公司 U-3010 型 紫外可见分光光度计)、苂光光谱(Hitachi 公司 F-4600 型苂光光谱仪). 滴定所用金属离子 $\left(\mathrm{Na}^{+} 、 \mathrm{Mg}^{2+} 、 \mathrm{Ba}^{2+} 、\right.$ $\mathrm{Hg}^{2+} 、 \mathrm{Cu}^{2+} 、 \mathrm{Cr}^{3+} 、 \mathrm{Zn}^{2+} 、 \mathrm{Fe}^{2+} 、 \mathrm{Ni}^{2+} 、 \mathrm{Co}^{2+} 、 \mathrm{Cd}^{2+} 、$ $\mathrm{Ag}^{+}$等)均为高氯酸盐.

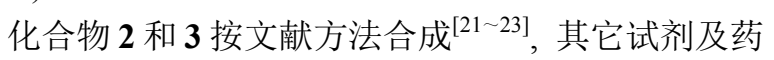
品(分析纯)购于国药集团化学试剂公司; 无水溶剂按文 献方法纯化制备, 其他试剂均为分析纯, 使用前未作进 一步纯化.

\section{2 实验方法}

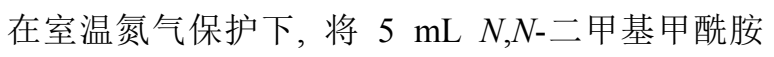

(DMF)、150 mg (0.46 mmol) 2,4,6-三-三叠氮乙基-1,3,5三甲基苯(2)和 $450 \mathrm{mg}(1.84 \mathrm{mmol})$ 化合物 $3(1.84 \mathrm{mmol})$ 加入到 $50 \mathrm{~mL}$ 的烧瓶中, 然后再将 $25 \mathrm{mg}(0.1 \mathrm{mmol})$ 五 水硫酸铜和 $30 \mathrm{mg}$ 抗坏血酸钠 $(0.15 \mathrm{mmol})$ 快速加入溶液 中, 在室温下搅拌反应 $3 \mathrm{~h}$. 反应结束后, 用水将其淬 灭, 并将悬浊液过滤, 收集其中的固体. 所得固体用柱 色谱层析纯化, 得到白色固体 $1272 \mathrm{mg}$, 产率 62\%. m.p. $115 \sim 116{ }^{\circ} \mathrm{C}$; ${ }^{1} \mathrm{H}$ NMR (400 MHz, DMSO- $d_{6}$ ) $\delta: 8.57$ (s, $3 \mathrm{H}), 8.23(\mathrm{dd}, J=3.2,6.3 \mathrm{~Hz}, 6 \mathrm{H}), 8.08(\mathrm{dd}, J=3.2,6.4$ $\mathrm{Hz}, 6 \mathrm{H}), 7.95$ (s, 3H), 7.46 (dd, $J=3.3,6.6 \mathrm{~Hz}, 12 \mathrm{H}), 5.63$ (s, 6H), 5.37 (s, 6H), 4.66 (s, 6H), $2.81(\mathrm{~d}, J=7.4 \mathrm{~Hz}, 6 \mathrm{H})$, $0.76(\mathrm{t}, J=7.2 \mathrm{~Hz}, 9 \mathrm{H}) ;{ }^{13} \mathrm{C}$ NMR $\left(100 \mathrm{MHz}\right.$, DMSO- $\left.d_{6}\right)$ $\delta: 146.2,131.3,130.8,130.4,129.22,128.4,126.6,125.6$, $124.8,124.3,63.8,63.5,47.9,23.5,15.5$; ESI-TOF-MS $m / z: 1066.5115(\mathrm{M}+1)^{+}$. Anal. calcd for $\mathrm{C}_{69} \mathrm{H}_{63} \mathrm{~N}_{9} \mathrm{O}_{3}$ : C 77.72, H 5.96, N 11.82; found C 77.49, H 6.00, N 11.78.

\section{3 紫外及荧光光谱滴定测试}

紫外及苂光光谱测定: 受体分子浓度为 $2 \times 10^{-5}$ $\mathrm{mol} \cdot \mathrm{L}^{-1}$ 的乙腈 - 水 $(V: V=9: 1)$ 溶液, 金属离子配制成 $2 \times 10^{-3} \mathrm{~mol} / \mathrm{L}$ 溶液.

荧光光谱测定: 激发波长为 $360 \mathrm{~nm}$. 测试时用微型 移液器向 1 中滴加, 保持受体分子的浓度基本不变. 利 用滴加法逐次加入高氯酸盐的阳离子, 观察受体分子与 阳离子结合后紫外及苂光光谱变化.

\section{$3.4{ }^{1} \mathrm{H}$ NMR 滴定测试}

Bruker Vance $400 \mathrm{MHz}$ 核磁共振仪, $\mathrm{CD}_{3} \mathrm{CN} / \mathrm{CDCl}_{3}$ 溶液化合物 $1\left(2.0 \times 10^{-4} \mathrm{~mol} / \mathrm{L}\right)$ 中利用累积滴加法逐次 加入高氯酸盐的阳离子, 观察受体分子与阳离子结合后 活泼氢化学位移的变化.

辅助材料(Supporting Information) 化合物 $\mathbf{1}$ 的 ${ }^{1} \mathrm{H}$ NMR、 ${ }^{13} \mathrm{C}$ NMR. ESI-MS 图谱. 这些材料可以免费从 
本刊网站(http://sioc-journal.cn/)上下载.

\section{References}

[1] Renzoni, A.; Zino, F.; Franchi, E. Environ. Res. 1998, 77, 68.

[2] Dorea, J. G.; Donangelo, C. M. Clin. Nutr. 2006, 25, 369.

[3] Nolan, E. M.; Lippard, S. J. Chem. Rev. 2008, 108, 3443.

[4] Kim, H. N.; Ren, W. X.; Kim, J. S.; Yoon, J. Chem. Soc. Rev. 2012, 41, 3210.

[5] Yang, Y.; Zhao, Q.; Feng, W.; Li, F. Chem. Rev. 2013, 113, 192.

[6] Pola, M. K.; Raju, M. V. R.; Lin, C. M.; Putikam, R.; Lin, M. C.; Epperla, C. P.; Chang, H. C.; Chen, S. Y.; Lin, H. C. Dyes Pigm. 2016, 130, 256

[7] Jiao, Y.; Zhang, L.; Zhou, P. Talanta 2016, 150, 14.

[8] Wan D.; Li Y.; Zhu P. Sens. Actuators, B 2015, 221, 1271.

[9] Cheng, H.; Qian, Y. Sens. Actuators, B 2015, 219, 57.

[10] Hemamalini, A.; Das, T. M. New J. Chem. 2013, 37, 2419.

[11] Hu, B.; Hu, L.-L.; Chen, M.-L.; Wang, J.-H. Biosens. Bioelectron. 2013, 49, 499

[12] Kumari, N.; Dey, N.; Bhattacharya, S. Analyst 2014, 139, 2370.

[13] Li, M.; Li, X. J.; Lu, H. Y.; Chen, C. F. Sens. Actuators, B 2014, 202,583 .

[14] Li, M.; Lu, H. Y.; Liu, R. L.; Chen, J. D.; Chen, C. F. J. Org. Chem. 2012, 77, 3670 .

[15] Hu, S. Z.; Chen, C. F. Org. Biomol. Chem. 2011, 9, 5838.

[16] Li, G. K.; Liu, M.; Yang, G. Q.; Chen, C. F.; Huang, Z. T. Chin. J. Chem. 2008, 26, 1440.

[17] Chen, Q. Y.; Chen, C. F. Tetrahedron Lett. 2005, 46, 165.

[18] Cao, Q.-Y.; Han, Y.-M.; Wang, H.-M.; Xie, Y. Dyes Pigm. 2013, 99, 798.

[19] Vedamalai, M.; Kedaria, D.; Vasita, R.; Mori, S.; Gupta, I. Dalton Trans. 2016, 45, 2700.

[20] Singh, R. S.; Gupta, R. K.; Paitandi, R. P.; Misra, A.; Pandey, D. S. New J. Chem. 2015, 39, 2233.

[21] Neupane, L. N.; Kim, J. M.; Lohani, C. R.; Lee K. H. J. Mater. Chem. 2012, 22, 4003.

[22] Shi, W.-J.; Liu, J.-Y.; Ng, D. K. P. Chem. Asian J. 2012, 7, 196.

[23] Dai, B.-N.; Cao, Q.-Y.; Wang, L.; Wang, Z.-C.; Yang, Z. Inorg.
Chim. Acta 2014, 423, 163.

[24] Ge, J.-Z.; Zou, Y.; Yan, Y.-H.; Lin, S.; Zhao, X.-F.; Cao, Q.-Y. J. Photochem. Photobiol. A: Chem. 2016, 315, 67.

[25] Xiong, X.; Cai, L.; Tang, Z. Chin. J. Org. Chem. 2012, 32, 1410 (in Chinese).

(熊兴泉, 蔡雷, 唐忠科, 有机化学, 2012, 32, 1410.)

[26] Rostovtsev, V. V.; Green, L. G.; Fokin, V. V.; Sharpless, K. B. Angew. Chem., Int. Ed. 2002, 41, 2597.

[27] Wang, Z.; Dai, B.; Qiu, J.; Cao, Q.; Ge, J. Chin. J. Org. Chem. 2015, 35, 2383 (in Chinese).

(王智成，戴博娜，丘继芳，曹迁永，葛金柱，有机化学，2015, 35,2383 .)

[28] Nie, J.; Li, J.; Deng, H.; Pan, H. Chin. J. Anal. Chem. 2015, 43, 609 (in Chinese). (聂䩀, 李建平, 邓欢, 潘宏程, 分析化学, 2015, 43, 609.)

[29] Lau, Y. H.; Rutledge, P. J.; Watkinson, M.; Todd, M. H. Chem. Soc. Rev. 2011, 40, 2848.

[30] Kuswandi, B.; Verboom, W.; Reinhoudt, D. N. Sensors 2006, 6, 978.

[31] Schweinfurth, D.; Krzystek, J.; Schapiro, I.; Demeshko, S.; Klein, J.; Telser, J.; Ozarowski, A.; Su, C.-Y.; Meyer, F.; Atanasov, M.; Neese, F.; Sarkar, B. Inorg. Chem. 2012, 51, 7592.

[32] Connell, T.-U.; Schieber, C.; Silvestri, I.-P.; White, J.-M.; Williams, S.-J.; Donnelly, P.-S. Inorg. Chem. 2014, 53, 6503.

[33] Meldal, M.; Tornøe, C. W. Chem. Rev. 2008, 108, 2952.

[34] Ingale, S. A., Seela, F. J. Org. Chem. 2012, 77, 9352.

[35] Baschieri, A.; Mazzanti, A.; Stagni, S.; Sambri, L. Eur. J. Inorg. Chem. 20132432.

[36] González, M. del C.; Otón, F.; Espinosa, A.; Tárraga, A.; Molina, P. Org. Biomol. Chem. 2015, 13, 1429.

[37] Zhu, J.-H.; Fan, X.-T.; Cao, Q.-Y. Inorg. Chim. Acta 2016, 449, 31.

[38] Vacca, A.; Nativi, C.; Cacciarini, M.; Pergoli, R.; Roelens, S. J. Am. Chem. Soc. 2004, 126, 16456.

[39] Wallace, K. J.; Hanes, R.; Anslyn, E.; Morey, J.; Kilway, K. V.; Siegel, J. Synthesis 2005, 2080.

[40] Benesi, H.-A.; Hildebrand, J.-H. J. Am. Chem. Soc. 1949, 71, 2703.

[41] Cao, Q.-Y.; Wang, Z.-C.; Li, M.; Liu, J.-H. Tetrahedron Lett. 2013, $54,3933$. 